\title{
Epiglottis cross-sectional area and oropharyngeal airway length in male and female obstructive sleep apnea patients
}

\author{
Melinda A Ma' \\ Rajesh Kumar ${ }^{2-4}$ \\ Paul M Macey ${ }^{4,5}$ \\ Frisca L Yan-Go6 \\ Ronald M Harper ${ }^{1,4}$ \\ 'Department of Neurobiology, \\ ${ }^{2}$ Department of Anesthesiology, \\ ${ }^{3}$ Department of Radiological Sciences, \\ David Geffen School of Medicine, \\ ${ }^{4}$ Brain Research Institute, ${ }^{5} \mathrm{UCLA}$ \\ School of Nursing, ${ }^{6}$ Department of \\ Neurology, David Geffen School of \\ Medicine, University of California at \\ Los Angeles, Los Angeles, CA, USA
}

Correspondence: Ronald M Harper Department of Neurobiology, David Geffen School of Medicine at UCLA, University of California, 10833 Le Conte Avenue, Los Angeles, CA 90095-1763, USA

Tel + I 3108255303

Fax +I 3108252224

Email rharper@ucla.edu
This article was published in the following Dove Press journal:

Nature and Science of Sleep

3 October 2016

Number of times this article has been viewed

Introduction: Obstructive sleep apnea (OSA) is a male-predominant condition, characterized by repeated upper-airway collapse with continued diaphragmatic efforts during sleep, and is accompanied by severe physiological consequences. Multiple morphological aspects, including epiglottis cross-sectional area (CSA) and oropharyngeal airway length (OPAL), can contribute to airway collapsibility in the condition. This study focused on the effects of OSA severity, sex, and race on OPA dimensions.

Materials and methods: Two high-resolution $T_{1}$-weighted image series were collected from 40 mild-to-severe OSA subjects (age $46.9 \pm 9$ years, body mass index $30.4 \pm 5.4 \mathrm{~kg} / \mathrm{m}^{2}$, ApneaHypopnea Index score $32.8 \pm 22.5,28$ males) and 54 control subjects ( $47 \pm 9$ years, $24.7 \pm 3.8 \mathrm{~kg} / \mathrm{m}^{2}$, 32 males) using a $3 \mathrm{~T}$ magnetic resonance-imaging scanner. Caucasian, Asian, African-American, and "other" subjects constituted the study pool. Both image series were realigned and averaged, and reoriented to a common space. CSA and OPAL were measured, normalized for subject height, and compared between sexes and disease-severity levels in OSA and control subjects. Results: Significantly reduced epiglottis CSA appeared only in severe OSA vs controls $(P=0.009)$. OPAL increased significantly with OSA severity vs controls (mild, $P=0.027$; moderate, $P<0.001$; severe, $P<0.001$ ). OSA males showed increased CSA and greater OPAL than OSA females, which may underlie the increased proportion of affected males with higher apnea-hypopnea index scores. However, no significant differences appeared between CSA and OPAL measures for male and female controls, suggesting that airway morphology may not be the sole contributor for airway collapse. No ethnic or racial differences appeared for CSA or OPAL measures.

Conclusion: Sex-based reductions in epiglottis CSA and increased OPAL in OSA subjects may enhance airway-collapse vulnerability, more so with greater disease severity, and partially underlie male vs female susceptibility to the sleep disorder.

Keywords: magnetic resonance imaging, apnea-hypopnea index, upper-airway length, gender, race

\section{Introduction}

Obstructive sleep apnea (OSA) affects approximately $10 \%$ of the adult population, and is characterized by repetitive upper-airway collapses during sleep with concomitant loss of airflow. ${ }^{1}$ The autonomic, physiologic, and neurophysiological consequences of OSA are severe, and mandate understanding of mechanisms underlying the genesis and maintenance of the condition.

The morphology of upper-airway structures plays a major role in OSA pathogenesis. Although reduced contraction forces of upper-airway dilators restrict airway dimensions, 
local anatomical characteristics, such as airway narrowness and elongation or impaired innervation, contribute to the disorder. $^{2-4}$ These aspects combine with gross anatomical features that accelerate airflow through a narrowed airway path, resulting in airway collapse from negative pharyngeal pressure. ${ }^{5,6}$ Approximately $12 \%$ of OSA patients undergo airway collapse at the epiglottis; however, the circumstances surrounding processes in epiglottis airway collapse remain only partially understood. ${ }^{7}$

The human pharynx is unsupported by rigid structures, except at its upper (bony) and lower (cartilage) extremities. ${ }^{5}$ Therefore, the unsupported pharyngeal tissues are largely susceptible to airway collapse under negative pressures generated during inspiration with a diminished airway. ${ }^{8}$ The most common feature used to differentiate airway morphology between OSA from control subjects is the minimum cross-sectional surface area of the oropharynx. ${ }^{2,9,10}$ The oropharyngeal airway (OPA) consists of the retropalatal region, extending from the hard palate to the caudal border of the soft palate, and the retroglossal region, extending from the caudal border of the soft palate to the base of the epiglottis, and is principally comprised of pharyngeal muscles, with little bone structure for maintaining rigidity., ${ }^{5,11}$ The majority of occlusions in OSA subjects occur in the retropalatal region. ${ }^{5,11}$ However, morphological characteristics of the retroglossal region of the oropharynx may also contribute to obstruction. The extent of cross-sectional area (CSA) between OSA and control subjects at the tip of the epiglottis, and the OPA length (OPAL; the distance between the hard palate and the tip of the epiglottis) remain unclear, especially the dimensions between male and female OSA and controls. These dimensions are important, as both lumen size and length determine collapsibility of the airway, and likely differ by sex.

Men are at increased risk of sleep apnea over women, possibly a consequence of upper-airway morphology and effects of those airway-dimension differences on airflow restriction. The prevalence of adult OSA is almost three times higher in males compared to premenopausal females. ${ }^{12-14}$ The upper-airway anatomy issues are of particular interest, as women with OSA tend to be more obese and have greater fat mass and body mass index (BMI) than men with OSA. ${ }^{15}$ The distribution of fat in men, preferentially appearing around cervical regions over a greater number of peripheral sites than in women, and fundamental sex-related differences in pharyngeal anatomy may predispose men to increased upper-airway collapsibility. ${ }^{12,15}$ Sex-related differences in OSA subjects appear in neck soft-tissue size and
CSA. Upper-airway length is also greater in control males than females, notably at postpuberty and adulthood, further supporting male predisposition for airway collapse. ${ }^{12,16}$ Other risk factors associated with OSA are obesity and race. ${ }^{17}$ This study examined BMI and multiple racial categories (Caucasian, Asian, African-American, and "other") in accordance with sex and OSA-severity effects on CSA and OPAL. The objective was to determine whether anatomical features of airway dimensions in OSA differ from those of controls, and whether particular aspects of airway morphology differ by OSA severity, sex, and race.

\section{Materials and methods Subjects}

We studied 40 OSA (age $46.9 \pm 9$ years, BMI $30.4 \pm 5.4 \mathrm{~kg} / \mathrm{m}^{2}$, 28 males) and 54 control subjects ( $47 \pm 9$ years, $24.7 \pm 3.8 \mathrm{~kg}$ / $\mathrm{m}^{2}, 32$ males). Racial categories were Caucasian, Asian, African-American, and "other" (Hispanic and mixed races). Our study included 43 Caucasian patients ( 25 controls, 18 OSA), 28 Asians (18 controls, ten OSA), nine African-Americans (five controls, four OSA), and 14 "other". Thirteen patients (nine controls and four OSA) had undergone tonsillectomies as a child. Only two OSA patients had a history of asthma or allergies. We considered the proportion of patients with a history of asthma or allergies to be too small to affect the outcome. There were no recorded tonsil scores for control or OSA subjects. All subjects had complete magnetic resonance imaging (MRI) of the upper airway with sufficient resolution for measures. All OSA subjects were newly diagnosed via overnight polysomnography and recruited from an accredited sleep-disorder laboratory at the University of California at Los Angeles (UCLA) Medical Center. OSA severity in subjects was classified based on the 1999 criteria from the American Academy of Sleep Medicine. ${ }^{18}$

The apnea-hypopnea index (AHI) is a severity index of sleep-disordered breathing, and includes both apneas and hypopneas; the index is derived by dividing the number of apnea and hypopnea events by the total sleep time. OSA subjects were categorized as follows: mild OSA, AHI of 5 or more, but fewer than 15 events/hour; moderate OSA, AHI of 15 or more, but fewer than 30 events/hour; and severe OSA, AHI of 30 or more events/hour. Of 40 OSA subjects, six showed mild OSA, 16 moderate OSA, and 18 severe OSA.

These subjects were part of a larger study, and OSA and control subjects included here were matched for similar agerange and precise MRI-scanning parameters, resulting in 40 OSA and 54 control subjects. None of the OSA subjects 
was taking any cardiovascular-altering medications, such as $\beta$-blockers, $\alpha$-agonists, angiotensin-converting enzyme inhibitors, or vasodilators, or mood-altering drugs such as serotonin-reuptake inhibitors. All control subjects were interviewed, with their sleep partner when possible, to rule out any undetermined OSA condition; subjects were referred for an overnight polysomnography if such a condition was suspected. Control subjects were healthy, had no history of cardiovascular or neurological defects, and were not taking any medications. We excluded all subjects with irremovable metals, such as pacemakers or stents. All subjects gave informed written consent prior to the study, and the study protocol was approved by the Institutional Review Board at UCLA.

\section{Sleep quality and daytime sleepiness}

All control and OSA subjects were assessed for sleep quality using the Pittsburgh Sleep Quality Index and daytime sleepiness using the Epworth Sleepiness Scale. These self-administered questionnaires were administered either immediately before or after MRI scanning.

\section{MRI}

The upper airway was examined using a $3 \mathrm{~T}$ MRI scanner (Magnetom Tim Trio; Siemens AG, Munich, Germany) with high-resolution $T_{1}$-weighted imaging procedures. All subjects were in an awake state, with the body in a supine position, and the head was fixed prior to scanning with foam pads placed on either side to minimize motion during data collection. Tongue position was standardized by maintaining all subjects alert and awake, with regular breathing. Patients were told not to swallow throughout the scanning process of approximately 6 minutes.

High-resolution $T_{1}$-weighted imaging was performed using a magnetization-prepared rapid-acquisition gradientecho pulse sequence (repetition time 2,200 ms, echo time $2.2 \mathrm{~ms}$, inversion time $900 \mathrm{~ms}$, flip angle $9^{\circ}$, matrix size $256 \times 256$, field of view $230 \times 230 \mathrm{~mm}$, slice thickness $1 \mathrm{~mm}$ ). The parallel-imaging technique, generalized autocalibrating partially parallel acquisition, with an acceleration factor of two was used for data acquisition.

\section{Data processing and analysis}

We used the statistical parametric mapping package SPM8, (http://www.fil.ion.ucl.ac.uk/spm), MRIcroN (http://people. cas.sc.edu/rorden/mricron/index.html), and MatLab-based (http://www.mathworks.com) custom-written software for data processing and analyses. Both high-resolution
$T_{1}$-weighted image series were realigned, averaged, and reoriented to position the cervical column for consistent assessment of surrounding soft-tissue measurement. The reoriented images of all OSA and control subjects were used for regional tracing of epiglottis CSA and OPAL assessment. All subjects were reviewed for lingual tonsil size, and none showed signs of enlargement or inflammation.

We identified landmarks at the midsagittal axis: hard palate, epiglottis tip, and midsagittal C2 vertebrae. The length of the oropharynx (hard palate to tip of the epiglottis) and the CSA at the tip of the epiglottis were then manually outlined. We computed CSAs (in $\mathrm{mm}^{2}$ ) using a MatLab-based program, which counted the number of pixels in the region of interest and multiplied by pixel area. OPALs (in mm), the distance from the hard palate to the tip of the epiglottis, were analyzed on midsagittal views by manually counting the number of pixels and multiplying by pixel length. Airway lengths were normalized for body height by using the OPAL:body height ratio $(\mathrm{mm} / \mathrm{meter})$ to ensure that observed differences were independent of sex and height.

\section{Statistical analysis}

SPSS version 20 (IBM, Armonk, New York) was used for data analyses. Demographic (age, sex, race) and biophysical (BMI) factors, and OPA morphology (CSA, OPAL) were assessed between severity- and sex-specific OSA and control subjects using one-way analysis of variance. All parametric values are expressed as mean \pm standard deviation for each group, and statistical significance level was set at $P<0.05$ for all variables.

\section{Results}

\section{Demographic and sleep variables}

Demographic, biophysical, and sleep variables of OSA and control subjects are summarized in Table 1. No significant differences in age $(P=0.93)$ appeared between control and

Table I Demographic, biophysical, and sleep data

\begin{tabular}{llll}
\hline Variables & $\begin{array}{l}\text { Controls (A), } \\
\text { mean } \pm \text { SD }\end{array}$ & $\begin{array}{l}\text { OSA (B), } \\
\text { mean } \pm \text { SD }\end{array}$ & $\begin{array}{l}\text { P-value, } \\
\text { A vs B }\end{array}$ \\
\hline Sex (male:female) & $32: 22$ & $28: 12$ & $<0.00 I^{*}$ \\
Age (years) & $47.0 \pm 9$ & $46.9 \pm 9$ & 0.928 \\
BMI $\left(\mathrm{kg} / \mathrm{m}^{2}\right)$ & $24.7 \pm 3.8$ & $30.4 \pm 5.4$ & $<0.00 I^{*}$ \\
AHI & - & $32.8 \pm 22.5$ & - \\
PSQI & $3.7 \pm 2.5$ & $9.1 \pm 4.1$ & $<0.00 I^{*}$ \\
ESS & $5.7 \pm 3.5$ & $10.4 \pm 4.9$ & $<0.00 I^{*}$ \\
\hline
\end{tabular}

Note: * indicates significant $P$-values.

Abbreviations: SD, standard deviation; OSA, obstructive sleep apnea; BMI, body mass index; AHI, apnea-hypopnea index; PSQI, Pittsburgh Sleep Quality Index; ESS, Epworth Sleepiness Scale. 
Table 2 Effects of race on airway dimensions

\begin{tabular}{|c|c|c|c|c|c|}
\hline Variables & Caucasian (A), $n=43$ & Asian (B), $n=28$ & $\begin{array}{l}\text { African-American (C), } \\
n=9\end{array}$ & Other (D), $n=14$ & $P$-value \\
\hline \multicolumn{6}{|l|}{ Controls } \\
\hline Height (feet) & $5.8 \pm 0.4$ & $5.3 \pm 0.3$ & $5.8 \pm 0.4$ & $5.6 \pm 0.4$ & $0.003^{*}$ \\
\hline Epiglottis cross-sectional area $\left(\mathrm{mm}^{2}\right)$ & $259.4 \pm 80.3$ & $269.6 \pm 92.4$ & $295.1 \pm 49$ & $251.3 \pm 121$ & 0.83 \\
\hline Normalized airway length (mm/meter) & $27.8 \pm 3.3$ & $27.8 \pm 4.2$ & $25.7 \pm 2$ & $28.5 \pm 2.8$ & 0.58 \\
\hline \multicolumn{6}{|l|}{ Obstructive sleep apnea } \\
\hline Height (feet) & $5.7 \pm 0.3$ & $5.7 \pm 0.3$ & $5.8 \pm 0.4$ & $5.5 \pm 0.2$ & 0.21 \\
\hline Epiglottis cross-sectional area $\left(\mathrm{mm}^{2}\right)$ & $220.3 \pm 95.9$ & $273.0 \pm 90.6$ & $223.0 \pm 139.0$ & $197.4 \pm 80.5$ & 0.39 \\
\hline Normalized airway length (mm/meter) & $32.5 \pm 3.2$ & $33.6 \pm 3.5$ & $29.2 \pm 2.4$ & $33.7 \pm 4.2$ & 0.15 \\
\hline
\end{tabular}

Note: * indicates significant $\mathrm{P}$-values.

Table 3 Area and length effects of OSA with increasing severity vs control subjects

\begin{tabular}{|c|c|c|c|c|c|c|c|}
\hline Variables & $\begin{array}{l}\text { Controls (A), } \\
n=54,32 \text { male }\end{array}$ & $\begin{array}{l}\text { Mild OSA (B), } \\
n=6,2 \text { male }\end{array}$ & $\begin{array}{l}\text { Moderate OSA (C), } \\
(n=\mid 6 ; \text { I I male) }\end{array}$ & $\begin{array}{l}\text { Severe OSA (D), } \\
n=18,15 \text { male }\end{array}$ & $\begin{array}{l}\text { P-value } \\
\text { (A vs B) }\end{array}$ & $\begin{array}{l}P \text {-value } \\
\text { (A vs C) }\end{array}$ & $\begin{array}{l}\text { P-value } \\
\text { (A vs D) }\end{array}$ \\
\hline Age (years) & $47 \pm 9$ & $54 \pm 7.2$ & $44.5 \pm 8.7$ & $46.6 \pm 9$ & 0.071 & 0.323 & 0.844 \\
\hline BMI $\left(\mathrm{kg} / \mathrm{m}^{2}\right)$ & $24.7 \pm 3.8$ & $28.4 \pm 6.6$ & $29.3 \pm 4.5$ & $32 \pm 5.5$ & 0.055 & $0.001 *$ & $<0.00 I^{*}$ \\
\hline $\mathrm{AHI}$ & - & $8.9 \pm 3.2$ & $20.4 \pm 4$ & $51.7 \pm 20.7$ & - & - & - \\
\hline $\begin{array}{l}\text { Epiglottis cross- } \\
\text { sectional area } \\
\left(\mathrm{mm}^{2}\right)\end{array}$ & $265.2 \pm 85.8$ & $255.3 \pm 142.1$ & $252 \pm 72.1$ & $200.1 \pm 95.7$ & 0.798 & 0.605 & $0.009 *$ \\
\hline $\begin{array}{l}\text { Normalized airway } \\
\text { length }(\mathrm{mm} / \mathrm{m})\end{array}$ & $27.7 \pm 3.5$ & $31.1 \pm 4.1$ & $32.4 \pm 2.2$ & $33.6 \pm 4.2$ & $0.027^{*}$ & $<0.001 *$ & $<0.00 I^{*}$ \\
\hline
\end{tabular}

Note: * indicates significant $P$-values.

Abbreviations: OSA, obstructive sleep apnea; BMI, body mass index; $\mathrm{AHI}$, apnea-hypopnea index.

OSA groups. There were 19 obese OSA patients (BMI range $30-43.2 \mathrm{~kg} / \mathrm{m}^{2}$ ) and 16 overweight OSA patients (BMI range, $25.2-29.2 \mathrm{~kg} / \mathrm{m}^{2}$ ) of a total of 40 OSA subjects. Three control subjects were considered obese, and 24 controls were overweight. There were significant differences in sex $(P<0.001)$, BMI $(P<0.001)$, Pittsburgh Sleep Quality Index $(P<0.001)$, and Epworth Sleepiness Scale $(P<0.001)$. No significant differences were found between racial groups for CSA (control, $P=0.83$; OSA, $P=0.39$ ) or OPAL (control, $P=0.58$; OSA, $P=0.15)$. Effects of race and height on airway dimensions are detailed in Table 2.

\section{Control and OSA dimensions with increasing OSA severity}

Values for epiglottis CSA and normalized OPAL of OSA with increasing severity and control subjects are shown in Table 3. Male and female measurements were combined within the groups of control, mild OSA, moderate OSA, and severe OSA when performing statistical analyses. Mean age was comparable between the OSA and control subjects. Moderate and severe OSA subjects significantly differed in BMI over controls (moderate, $P=0.001$; severe, $P<0.001$ ). As expected, OSA patients had a BMI close to the obesity criteria of 30 (moderate, 29.3 \pm 4.5 ; severe, 32 \pm 5.5 ).

CSA was determined in the midsagittal plane at the level of the epiglottis tip. Significant reductions in epiglottis CSA area appeared in severe OSA vs controls $(P=0.009)$. Declines in CSA for mild and moderate OSA subjects appeared, but did not reach significance. Representative axial and sagittal MRI images of an OSA and control subject at the level of the epiglottis show significant reductions in CSA, as shown in Figure 1. OPAL, normalized for body height, increased significantly in each OSA-severity group vs control (mild, $P=0.027$; moderate, $P<0.001$; severe, $P<0.001)$. Sagittal MRI images of OPAL from one moderate OSA, one severe OSA, and one control subject are shown in Figure 2.

\section{Male-female differences}

The effects of sex on epiglottis CSA and OPAL are shown in Table 4. The study sample included a total of 60 males and 34 females: 28 OSA males and 12 OSA females, and 32 control males and 22 control females. There were no significant differences in age between men and women. The severity of OSA was higher in males than in females, despite a significantly lower BMI in OSA males than OSA females $(P=0.018)$.

When comparing sex differences, OPAL values were normalized for body height by using the OPAL:height ratio $(\mathrm{mm} / \mathrm{m})$. Adult male and female OSA subjects showed significantly longer OPAL than male and female controls, respectively (males, $P<0.001$; females, $P=0.001$ ). Significant differences in epiglottis CSA and OPAL appeared between 


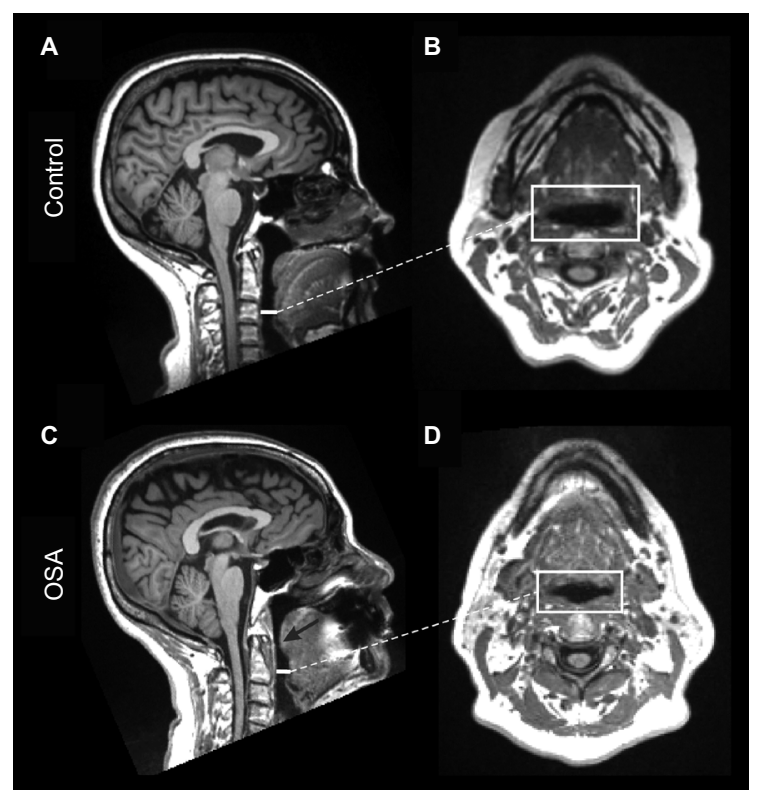

Figure I Reduced epiglottis cross-sectional area in an OSA subject over a control subject.

Notes: Each pair of MRI scans shows images obtained from the level of the dorsal tip of the epiglottis during wakefulness. Control subject (A) midsagittal MRI image of airway, and (B) axial image at the epiglottis level. Measures of cross-sectional area were taken at the horizontal white lines in images $(\mathbf{A}, \mathbf{C})$. OSA subject $(\mathbf{C})$ midsagittal MRI image shows narrowing (arrow) of pharyngeal upper airway at an elongated soft palate, and (D) axial image shows representative reduced crosssectional area at the epiglottis level. The cross-sectional areas corresponding to the horizontal lines are outlined in tracings (B, D).

Abbreviations: OSA, obstructive sleep apnea; MRI, magnetic resonance imaging. male and female OSA subjects (CSA, $P=0.016$; OPAL, $P=0.017)$. No significant differences emerged between male and female controls for CSA $(P=0.19)$ or OPAL $(P=0.058)$. Midsagittal MRI images of OPAL for male and female control and OSA subjects are depicted in Figure 3.

\section{Discussion}

This study investigated the association of epiglottis CSA and OPAL with OSA severity and sex in adult male and female control and OSA subjects. Only severe OSA subjects showed significant reduction in epiglottis CSA, possibly predisposing this airspace to collapse during sleep. OPAL was significantly lengthened in subjects with mild, moderate, or severe AHI. CSA and OPAL in OSA subjects differed with respect to sex, with OSA males having increased CSAs and longer OPALs than those of OSA females. No racial or ethnic differences appeared in the study measures. Male-female anatomical differences may underlie some of the sex-risk outcomes in the syndrome.

\section{Upper-airway morphology}

The human upper airway is prone to obstruction, because of nonrigid support of the pharyngeal musculature and paucity of

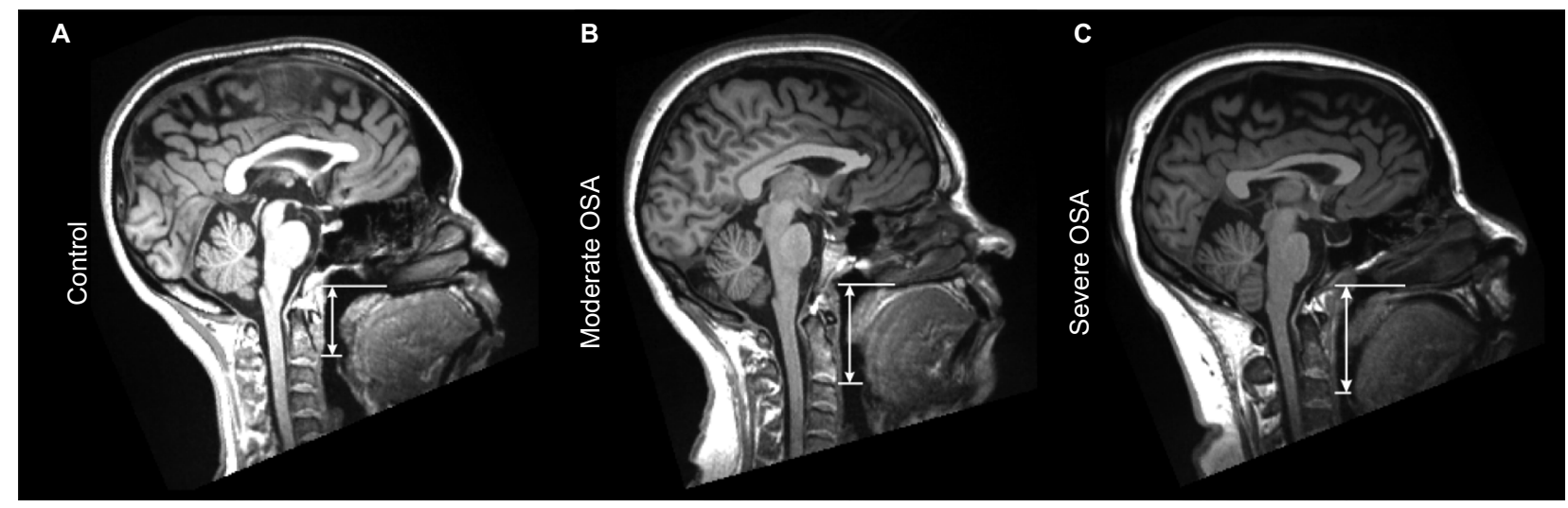

Figure 2 Increased oropharyngeal length in OSA subjects with differing severity vs a control subject.

Notes: Midsagittal MRI of oropharyngeal airway length during wakefulness, measured as the distance from the hard palate to the dorsal tip of the epiglottis, of a female control subject (A), female OSA subject with moderate $\mathrm{AHI}(\mathbf{B})$, and female OSA subject with severe $\mathrm{AHI}(\mathbf{C})$.

Abbreviations: OSA, obstructive sleep apnea; MRI, magnetic resonance imaging; AHI, apnea-hypopnea index.

Table 4 Area and length effects of sex and OSA

\begin{tabular}{|c|c|c|c|c|c|c|c|c|}
\hline Variables & $\begin{array}{l}\text { Male controls } \\
\text { (A), } n=32\end{array}$ & $\begin{array}{l}\text { Female controls } \\
\text { (B), } n=22\end{array}$ & $\begin{array}{l}\text { Male OSA (C), } \\
n=28\end{array}$ & $\begin{array}{l}\text { Female OSA } \\
\text { (D), } n=12\end{array}$ & $\begin{array}{l}P \text {-value, } \\
A \text { vs B }\end{array}$ & $\begin{array}{l}P \text {-value, } \\
\text { A vs C }\end{array}$ & $\begin{array}{l}P \text {-value, } \\
\text { B vs D }\end{array}$ & $\begin{array}{l}P \text {-value, } \\
\text { C vs D }\end{array}$ \\
\hline Age (years) & $45.3 \pm 9.2$ & $49.5 \pm 8.3$ & $45 \pm 8.9$ & $51.3 \pm 8$ & 0.084 & 0.881 & 0.582 & $0.039 *$ \\
\hline BMI $\left(\mathrm{kg} / \mathrm{m}^{2}\right)$ & $25.1 \pm 2.6$ & $24 \pm 5.1$ & $29.3 \pm 4.9$ & $32.9 \pm 5.8$ & 0.366 & $0.001^{*}$ & $<0.001 *$ & $0.018 *$ \\
\hline $\mathrm{AHI}$ & - & - & $35.4 \pm 21.5$ & $26.7 \pm 24.8$ & - & - & - & 0.086 \\
\hline $\begin{array}{l}\text { Epiglottis cross- } \\
\text { sectional area }\left(\mathrm{mm}^{2}\right)\end{array}$ & $278.3 \pm 98$ & $246.2 \pm 61.2$ & $251.3 \pm 99.1$ & I $77.4 \pm 68.4$ & 0.19 & 0.238 & $0.031 *$ & $0.016 *$ \\
\hline $\begin{array}{l}\text { Normalized airway } \\
\text { length }(\mathrm{mm} / \mathrm{m})\end{array}$ & $28.4 \pm 3.7$ & $26.6 \pm 2.9$ & $33.6 \pm 3.6$ & $30.8 \pm 2.6$ & 0.058 & $<0.00 I^{*}$ & $0.001 *$ & $0.017 *$ \\
\hline
\end{tabular}

Note: $*$ indicates significant $P$-values.

Abbreviations: OSA, obstructive sleep apnea; BMI, body mass index; AHI, apnea-hypopnea index. 


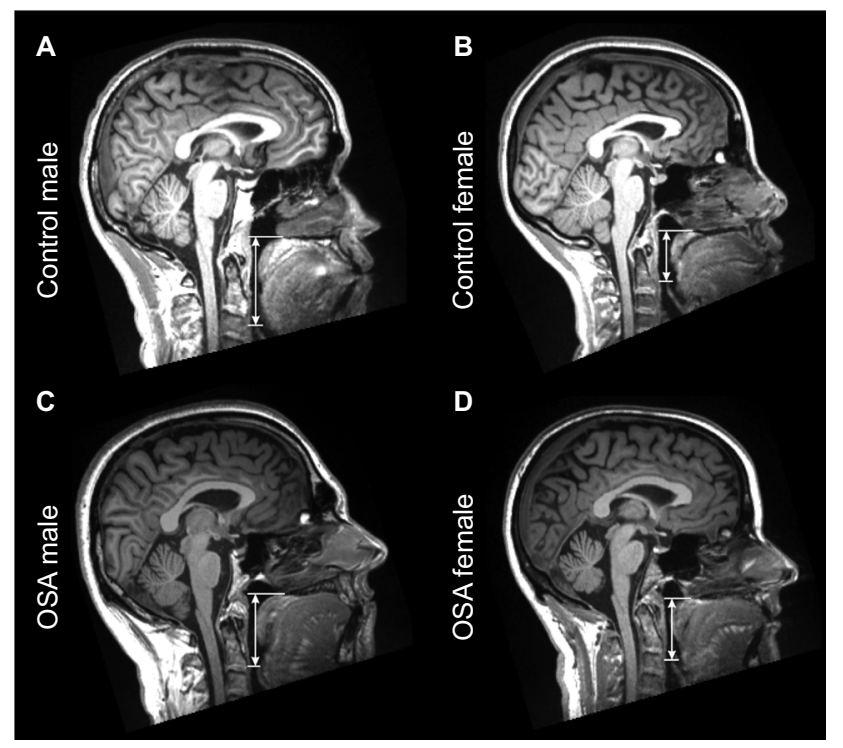

Figure 3 Increased oropharyngeal length in a male control and OSA subject vs a female control and OSA subject.

Notes: Midsagittal MRI of the OPAL of a male control subject (A) compared to a female control subject (B), and a male OSA subject (C) compared to a female OSA subject (D). Males displayed elongated OPAL over females.

Abbreviations: OSA, obstructive sleep apnea; MRI, magnetic resonance imaging; OPAL, oropharyngeal airway length.

bony or cartilaginous structures. ${ }^{5}$ Much of the airway above the cartilaginous larynx is susceptible to collapse, due to inflammatory swelling of tonsillar tissue, fat infiltration, or reduced upper-airway dilator-muscle activity, resulting in reduced airway size during inspiration. ${ }^{9,19,20}$ A smaller pharyngeal CSA decreases pressure within the airway by restricting airflow and accelerating airspeed, enhancing the potential for collapse. ${ }^{5,21}$ The oropharynx is the site of most concern for collapse, as it is the narrowest region of the upper airway., ${ }^{41,22}$

A longer upper airway also contributes to the potential for airway collapse in OSA patients. ${ }^{4}$ Increased OPAL has been associated with increased pharyngeal collapsibility and OSA severity. The enhanced susceptibility to collapse stems from analogous physical examples; any lengthening of a pliable tube would be more susceptible to collapse under negative airway pressure.

Patients with severe OSA had the narrowest CSA at the tip of the epiglottis. OPAL increased significantly for OSA patients with increasing apnea severity. A slight decline in CSA or increase in airway length may be functionally significant, and thus these structural differences may be important variables that impact pharyngeal collapsibility.

\section{Male-female differences}

Major risk factors for sleep-disordered breathing include male sex, aging, race, and obesity. ${ }^{17}$ This study considered racial differences in the statistical analyses, which confirmed that race did not contribute to different airway-morphology values between control and OSA subjects. Most OSA patients are obese, with at least $70 \%$ of morbidly obese patients suffering from OSA. ${ }^{23}$ Our study consisted of 19 obese OSA subjects and 16 overweight OSA subjects from a total of 40 OSA individuals. Three control subjects were considered obese, and 24 controls were considered overweight (BMI $\left.25-29.9 \mathrm{~kg} / \mathrm{m}^{2}\right)$.

Excess body-fat deposition with aging and obesity differs in character between the sexes, and may play a mechanistic role in upper-airway obstruction during sleep. ${ }^{12,24,25}$ Males have more predisposing factors of airway collapse than females, including more fat distribution in central body areas and longer upper-airway lengths, whereas females have fat distributed more in the hips and thighs. ${ }^{12,15,24}$ Male-female differences in fat distribution surrounding the collapsible portion of the pharynx may contribute to airflow characteristics, in addition to morphological variation. Sex differences in upper-airway anatomy may contribute to the male predominance of OSA in adults. An increase in airway length in males over females occurs during puberty, possibly from actions of testosterone or other hormones, and that increase enhances the potential for upper-airway collapsibility. ${ }^{12,15}$

Male OSA subjects had significantly larger CSAs than those of female OSA subjects, making pharyngeal area a less likely predisposing factor of airway collapse in affected males. Although OSA patients had smaller CSAs than controls, the greater tidal volumes in males require larger CSAs than those of females. The larger male CSA values may have been insufficiently large to overcome the enhanced airflow needs for men. Both male and female OSA subjects showed longer OPAL over male and female control subjects, respectively. Male OSA subjects showed a significant increase in OPAL over female OSA subjects, supporting previous findings by others. Our data suggest that female OSA patients may be equally susceptible as male counterparts for airway collapse with altered CSA and OPAL pharyngeal morphology.

A remarkable finding was that no significant differences appeared between CSA and normalized OPAL measures for male and female control subjects, an outcome that contrasts with earlier studies indicating that airway length is inherently longer in control males than females. ${ }^{12,16}$ When comparing sex effects, the diverse racial sample and high average BMI for both control and OSA subjects may have contributed to variability in CSA and OPAL measurements. The control subjects were borderline overweight (BMI $24.7 \pm 3.8 \mathrm{~kg} / \mathrm{m}^{2}$ ), which may explain the insignificant differences in CSA and 
OPAL between male and female controls. Prevalence of SA is greater in overweight or obese people and minority racial groups (Asians, African-Americans, Hispanics). ${ }^{26}$ Asians demonstrate differing craniofacial features, such as smaller maxilla and mandibular size, larger oropharyngeal CSA, and smaller facial height, compared to Caucasians. ${ }^{26}$ For future studies, race and ethnicity should be matched across control and OSA subjects, and comorbid medical conditions carefully partitioned, especially for conditions unique to minority populations, including obesity, which may enhance the prevalence of OSA.

The inherent sex-related morphological differences do not preclude the use of functional processes to reduce the potential for OSA. We speculate that airway vocalization exercises may provide such an intervention, considering the successful use of didgeridoo training to reduce OSA characteristics. ${ }^{27}$ Knowledge of the specific characteristics of upper-airway morphology between males and females is essential in determining appropriate upper-airway exercises to prevent airway collapse.

\section{Limitations}

Limitations in this study include a relatively small sample size for female OSA subjects compared to male OSA subjects ( 28 males and 12 females), reorientation accuracy of the MRI scans, and unavailability of complete MRI scans showing the total OPA reaching the caudal base of the epiglottis. Raw results for epiglottis CSA were used, without normalizing for anatomic variables, such as body surface area.

MRI scans of all subjects were performed exclusively during wakefulness. Upper-airway pharyngeal muscles contract actively during wakefulness, and may not fully correspond to airway dimensions of CSA and OPAL during sleep. ${ }^{28}$ Previous research has successfully demonstrated correlations between OSA severity and upper-airway morphology in the sleep state. ${ }^{29}$ By comparing CSA and OPAL during tidal breathing in wakefulness and during obstructive apnea in sleep, more accurate identification of the site of obstruction and complete pharyngeal wall movements during sleep can be obtained. ${ }^{10}$ Acquisition of images was not gated to the respiratory cycle, a limitation that may have affected structural measurements; however, the image collection required 6 minutes of acquisition time, which would incorporate many respiratory cycles and allow stable sampling of those measures. Although it is difficult to avoid subjective judgment in the measurement of pharyngeal airway areas and lengths, we attempted to avoid bias by using standardized MRI realignment and structural identification by one investigator blinded to the MRI parameters.

\section{Conclusion}

The CSA of the pharyngeal airway at the tip of the epiglottis is reduced in severe adult male and female OSA subjects over controls, but not in those with mild or moderate OSA. OPAL, normalized for body height, is greater in mild, moderate, and severe OSA subjects for both sexes. It is likely the case that both CSA and OPAL contribute to airway collapse. When sex effects were examined in OSA, increased OPAL was a major risk factor, and appears to contribute substantially to severity of OSA and the enhanced male prevalence. There appears to be little effect of race on the airway measures considered here.

The potential to intervene to overcome morphological aspects of excessive OPAL in OSA is limited, leaving only the possibility to enhance functional characteristics of upper-airway musculature to assist in airway integrity. That enhancement may be instituted by muscle retraining through specific exercises or by enhancing sensory input that normally restores upper-airway tone. The specific outcomes here provide essential knowledge on sex-based pharyngeal morphology in OSA.

\section{Acknowledgment}

This research was supported by the National Institutes of Health (R01 HL-113251 and R01 NR-015038).

\section{Disclosure}

The authors report no conflicts of interest in this work.

\section{References}

1. Young T, Peppard PE, Gottlieb DJ. Epidemiology of obstructive sleep apnea: a population health perspective. Am J Respir Crit Care Med. 2002;165:1217-1239.

2. Shellock FG, Schatz CJ, Julien P, et al. Occlusion and narrowing of the pharyngeal airway in obstructive sleep apnea: evaluation by ultrafast spoiled GRASS MR imaging. AJR Am J Roentgenol. 1992;158:1019-1024.

3. Boyd JH, Petrof BJ, Hamid Q, Fraser R, Kimoff RJ. Upper airway muscle inflammation and denervation changes in obstructive sleep apnea. $\mathrm{Am}$ J Respir Crit Care Med. 2004;170:541-546.

4. Daulatzai MA. The pharyngeal landscape: its length and breadth. J Sleep Res. 2009;18:483-484.

5. Dempsey JA, Veasey SC, Morgan BJ, O’Donnell CP. Pathophysiology of sleep apnea. Physiol Rev. 2010;90:55-56.

6. Brown EC, Cheng S, McKenzie DK, Butler JE, Gandevia SC, Bilston LE. Respiratory movement of upper airway tissue in obstructive sleep apnea. Sleep. 2013;36(7):1069-1076.

7. Torre C, Camacho M, Liu SY, Huon LK, Capasso R. Epiglottis collapse in adult obstructive sleep apnea: a systematic review. Laryngoscope. 2016;126:515-523.

8. Isono S, Feroah TR, Hajduk EA, Brant R, Whitelaw WA, Remmers JE. Interaction of cross-sectional area, driving pressure, and airflow of passive velopharynx. J Appl Physiol (1985). 1997;83:851-859.

9. Ogawa T, Enciso R, Shintaku WH, Clark G. Evaluation of cross-sectional airway configuration of obstructive sleep apnea. Oral Surg Oral Med Oral Pathol Oral Radiol Endod. 2007;103:102-108. 
10. Horner RL, Shea SA, McIvor J, Guz A. Pharyngeal size and shape during wakefulness and sleep in patients with obstructive sleep apnoea. O J Med. 1989;72:719-735.

11. Li Y, Ye J, Li T, et al. Anatomic predictors of retropalatal mechanical loads in patients with obstructive sleep apnea. Respiration. 2011;82: 246-253.

12. Ronen O, Malhotra A, Pillar G. Influence of gender and age on upper-airway length during development. Pediatrics. 2007;120: e1028-e1034.

13. Subramanian S, Jayaraman G, Majid H, Aguilar R, Surani S. Influence of gender and anthropometric measures on severity of obstructive sleep apnea. Sleep Breath. 2012;16:1091-1095.

14. Bixler EO, Vgontzas AN, Lin H, et al. Prevalence of sleep-disordered breathing in women: effects of gender. Am J Respir Crit Care Med. 2001;163:608-613.

15. Malhotra A, Carden K. The debate about gender differences in obstructive sleep apnea. Sleep Med. 2003;4:485-487.

16. Malhotra A, Huang Y, Fogel RB, et al. The male predisposition to pharyngeal collapse: importance of airway length. Am J Respir Crit Care Med. 2002;166:1388-1395.

17. Pinto S, Huang J, Tapia I, et al. Effects of race on upper airway dynamic function during sleep in children. Sleep. 2011;34:495-501.

18. [No authors listed]. Sleep-related breathing disorders in adults: recommendations for syndrome definition and measurement techniques in clinical research. Sleep. 1999;22:667-688.

19. Yucel A, Mehmet U, Haktanir A, Acar M, Fidan F. Evaluation of the upper airway cross-sectional area changes in different degrees of severity of obstructive sleep apnea syndrome: cephalometric and dynamic CT study. AJNR Am J Neuroradiol. 2005;26:2624-2629.
20. Eckert DJ, Malhotra A. Pathophysiology of adult obstructive sleep apnea. Proc Am Thorac Soc. 2008;5:144-153.

21. Walsh JH, Leigh MS, Paduch A, et al. Evaluation of pharyngeal shape and size using anatomical optical coherence tomography in individuals with and without obstructive sleep apnoea. J Sleep Res. 2008;17: 230-238.

22. Avrahami E, Solomonovich A, Englender M. Axial CT measurements of the cross-sectional area of the oropharynx in adults with obstructive sleep apnea syndrome. AJNR Am J Neuroradiol. 1996;17:1107-1111.

23. Zhao X, Lui Y, Gao Y. Three-dimensional upper-airway changes associated with various amounts of mandibular advancement in awake apnea patients. Am J Orthod Dentofacial Orthop. 2008;133:661-668.

24. Simpson L, Mukherjee S, Cooper MN, et al. Sex differences in the association of regional fat distribution with the severity of obstructive sleep apnea. Sleep. 2010;33:467-474.

25. Horner RL, Mohiaddin RH, Lowell DG, et al. Sites and sizes of fat deposits around the pharynx in obese patients with obstructive sleep apnoea and weight matched controls. Eur Respir J. 1989;2:613-622.

26. Punjabi NM. The epidemiology of adult obstructive sleep apnea. Proc Am Thorac Soc. 2008;5:136-143.

27. Shigeta Y, Enciso R, Ogawa T, Shintaku WH, Clark GT. Correlation between retroglossal airway size and body mass index in OSA and non-OSA patients using cone beam CT imaging. Sleep Breath. 2008; 12:347-352.

28. Ward CP, York KM, McCoy JG. Risk of obstructive sleep apnea lower in double reed wind musicians. J Clin Sleep Med. 2012;8:251-255.

29. Heo JY, Kim JS. Correlation between severity of sleep apnea and upper airway morphology: cephalometry and MD-CT study during awake and sleep states. Acta Otolaryngol. 2011;131:84-90.
Nature and Science of Sleep

\section{Publish your work in this journal}

Nature and Science of Sleep is an international, peer-reviewed, open access journal covering all aspects of sleep science and sleep medicine, including the neurophysiology and functions of sleep, the genetics of sleep, sleep and society, biological rhythms, dreaming, sleep disorders and therapy, and strategies to optimize healthy sleep. The manuscript

\section{Dovepress}

management system is completely online and includes a very quick and fair peer-review system, which is all easy to use. Visit http://www. dovepress.com/testimonials.php to read real quotes from published authors. 